\title{
Common fixed points for some generalized multivalued nonexpansive mappings in uniformly convex metric spaces
}

\author{
Worawut Laowang ${ }^{1,2}$ and Bancha Panyanak ${ }^{1,2^{*}}$
}

\author{
* Correspondence: \\ banchap@chiangmai.ac.th \\ 'Department of Mathematics, \\ Faculty of Science, Chiang Mai \\ University, Chiang Mai 50200, \\ Thailand \\ Full list of author information is \\ available at the end of the article
}

\author{
Abstract \\ Abkar and Eslamian (Nonlinear Anal. TMA, 74, 1835-1840, 2011) prove that if $K$ is a \\ nonempty bounded closed convex subset of a complete CAT(0) space $X, t: K \rightarrow K$ is \\ a single-valued quasi-nonexpansive mapping and $T: K \rightarrow K C(K)$ is a multivalued \\ mapping satisfying conditions $(E)$ and $\left(C_{\lambda}\right)$ for some $\lambda \in(0,1)$ such that $t$ and $T$ \\ commute weakly, then there exists a point $z \in K$ such that $z=t(z) \in T(z)$. In this \\ paper, we extend this result to the general setting of uniformly convex metric \\ spaces. Nevertheless, condition (E) of $T$ can be weakened to the strongly \\ demiclosedness of $/$ - $T$.
}

Keywords: generalized multivalued nonexpansive mapping, commuting mapping, common fixed point, uniformly convex metric space

\section{Introduction}

Let $K$ be a nonempty subset of a $\operatorname{CAT}(0)$ space $(X, d)$ (see Bridson and Haefliger [[1] for more details on this space). A mapping $t: K \rightarrow X$ is said to be nonexpansive if

$$
d(t(x), t(y)) \leq d(x, y) \text { for all } x, y \in K \text {. }
$$

A point $x \in K$ is called a fixed point of $t$ if $x=t(x)$. We shall denote by Fix $(t)$ the set of fixed points of $t$ : The mapping $t$ is said to be quasi-nonexpansive if

$$
d(t(x), y) \leq d(x, y) \text { for all } x \in K \text { and } y \in \operatorname{Fix}(t) .
$$

Fixed point theory in $\mathrm{CAT}(0)$ spaces was first studied by Kirk [2,3]. He showed that every nonexpansive (single-valued) mapping defined on a bounded closed convex subset of a complete $\mathrm{CAT}(0)$ space always has a fixed point. Since then the fixed point theory for single-valued and multivalued mappings in CAT(0) spaces has been rapidly developed and many of papers have appeared (see e.g., [4-14] and the references therein). It is worth mentioning that fixed point theorems in CAT(0) spaces (specially in $\mathbb{R}$ - trees) can be applied to graph theory, biology, and computer science (see e.g., [15-20]).

In 2005, Dhompongsa et al. [6] obtained a common fixed point result for a commuting pair of single-valued and multivalued nonexpansive mappings in $\operatorname{CAT}(0)$ spaces. Shahzad and Markin [14] studied an invariant approximation problem and provided sufficient conditions for the existence of $z \in K \subseteq X$ such that $d(z, y)=\operatorname{dist}(y, K)$ and $z$

(c) 2011 Laowang and Panyanak; licensee Springer. This is an Open Access article distributed under the terms of the Creative Commons Attribution License (http://creativecommons.org/licenses/by/2.0), which permits unrestricted use, distribution, and reproduction in any medium, provided the original work is properly cited. 
$=t(z) \in T(z)$ where $y \in X, t$ and $T$ are commuting nonexpansive mappings on $K$. Shahzad [21] also obtained common fixed point and invariant approximation results for $t$ and $T$, which are weakly commuting.

In 2008, Suzuki [22] introduced a condition on mappings, which is weaker than nonexpansiveness and stronger than quasi-nonexpansivemess and called it condition (C). He proved some interesting fixed point theorems and convergence theorems for such mappings in Banach spaces setting. Motivated by Suzuki's results, Garcia-Falset et al. [23] introduced two generalizations of condition $(C)$, namely, conditions $(E)$ and $\left(C_{\lambda}\right)$ and studied both the existence of fixed points and the asymptotic behavior of mappings satisfying such conditions. Recently, Abkar and Eslamian [4] proved that if $K$ is a nonempty bounded closed convex subset of a complete CAT(0) space $X, t: K \rightarrow K$ is a single-valued quasi-nonexpansive mapping and $T: K \rightarrow K C(K)$ is a multivalued mapping satisfying conditions $(E)$ and $\left(C_{\lambda}\right)$ for some $\lambda \in(0,1)$ such that $t$ and $T$ are weakly commuting, then there exists a point $z \in K$ such that $z=t(z) \in T(z)$. In this paper, we extend this result to the general setting of uniformly convex metric spaces in the sense of Goebel and Reich [24]. Nevertheless, condition (E) of $T$ can be weakened to the strongly demiclosedness of $I-T$. Since uniformly convex Banach spaces and CAT(0) spaces are uniformly convex metric spaces, then our results extend and improve the results in $[4,25,26,23,27]$ and many others.

\section{Preliminaries}

Let $(X, d)$ be a metric space. A geodesic path joining $x \in X$ to $y \in X$ is a map $c$ from a closed interval $[0, l] \subset \mathbb{R}$ to $X$ such that $c(0)=x, c(l)=y$, and $d\left(c(t), c\left(t^{\prime}\right)\right)=\left|t-\mathrm{t}^{\prime}\right|$ for all $t$, $t^{\prime} \in[0, l]$. The image $c([0, l])$ of $c$ is called a geodesic segment joining $x$ and $y$. When it is unique this geodesic segment is denoted by $[x, y]$. The space $(X, d)$ is said to be a geodesic space if every two points of $X$ are joined by a geodesic path, and $X$ is said to be uniquely geodesic if there is exactly one geodesic path joining $x$ and $y$ for each $x, y \in X$. A point $z \in$ $X$ belongs to the geodesic segment $[x, y]$ if and only if there exists $t \in[0,1]$ such that

$$
d(z, x)=t d(x, y) \text { and } d(z, y)=(1-t) d(x, y),
$$

and we will write $z=(1-t) x \oplus t y$ for simplicity. A subset $K$ of $X$ is said to be convex if $K$ includes every geodesic segment joining any two of its points.

Definition 2.1 In a geodesic space $(X, d)$, the metric $d: X \times X \rightarrow \mathbb{R}$ is said to be convex if for any $x, y, z \in X$, one has

$$
d(x,(1-t) y \oplus t z) \leq(1-t) d(x, y)+t d(x, z) \text { for all } t \in[0,1] .
$$

A geodesic space $(X, d)$ with convex metric is called uniformly convex ([24]) if for any $r>0$ and $\varepsilon \in(0,2]$ there exists $\delta \in(0,1]$ such that if $a, x, y \in X$ with $d(x, a) \leq r$, $d(y, a) \leq r$ and $d(x, y) \geq \varepsilon r$, then

$$
d\left(\frac{1}{2} x \oplus \frac{1}{2} y, a\right) \leq(1-\delta) r .
$$

A mapping $\eta:(0, \infty) \times(0,2] \rightarrow(0,1]$ providing such a $\delta:=\eta(r, \varepsilon)$ for a given $r>0$ and $\varepsilon \in(0,2]$ is called a modulus of uniform convexity (see [28]). Notice that this definition of uniform convex metric spaces is weaker than the one used in [29] because this modulus of convexity does depend on the two variables $r$ and $\varepsilon$ while it is assumed 
to depend only on $\varepsilon$ in [29]. The mapping $\delta$ is called monotone if for every fix $\varepsilon$ it decreases with respect to $r$. It is called lower semi-continuous from the right if for every fix $\varepsilon$ it is lower semi-continuous from the right with respect to $r$. Throughout this paper, we assume that all uniformly convex metric spaces have monotone or lower semi-continuous from the right moduli of uniform convexity.

Recall that a subset $K$ of a metric space $X$ is said to be (uniquely) proximinal if each point $x \in X$ has a (unique) nearest point in $K$. In [28], Kohlenbach and Leustean prove that every decreasing sequence of nonempty bounded closed convex subsets of a complete uniformly convex metric space has nonempty intersection. As a consequence of this, we obtain the following result (see [[30], page 4] for the proof).

Proposition 2.2 Every nonempty closed convex subset of a complete uniformly convex metric space is uniquely proximinal.

The following result is proved by Kaewcharoen and Panyanak [30] in a uniformly convex metric space in the sense of [29]. We can state the result in the sense of Definition 2.1 because the proof is similar to the one given in [30].

Lemma 2.3 Let $K$ be a convex subset of a uniformly convex metric space and $t: K \rightarrow$ $K$ a quasi-nonexpansive mapping whose fixed point set is nonempty. Then Fix $(t)$ is closed and convex.

The following method and results deal with the concept of asymptotic centers. Let $(X, d)$ be a metric space and let $\left\{x_{n}\right\}$ be a bounded sequence in $X$. For $x \in X$, we set

$$
r\left(x,\left\{x_{n}\right\}\right)=\limsup _{n \rightarrow \infty} d\left(x, x_{n}\right) .
$$

The asymptotic radius of $\left\{x_{n}\right\}$ is given by

$$
r\left(\left\{x_{n}\right\}\right):=\inf \left\{r\left(x,\left\{x_{n}\right\}\right): x \in X\right\},
$$

and the asymptotic center of $\left\{x_{n}\right\}$ is the set

$$
A\left(\left\{x_{n}\right\}\right):=\left\{x \in X: r\left(x,\left\{x_{n}\right\}\right)=r\left(\left\{x_{n}\right\}\right)\right\} .
$$

The following result is proved in [31].

Lemma 2.4 If $X$ be a complete uniformly convex metric space and $\left\{x_{n}\right\}$ be a bounded sequence in $X$, then $A\left(\left\{x_{n}\right\}\right)$ is a singleton.

A bounded sequence $\left\{x_{n}\right\}$ is called regular if $r\left(\left\{x_{n}\right\}\right)=r\left(\left\{x_{n_{k}}\right\}\right)$ for every subsequence $\left\{x_{n_{k}}\right\}$ of $\left\{x_{n}\right\}$. It is known that in a Banach space, every bounded sequence always has a regular subsequence (see e.g., [[32], Lamma 15.2]). Since the proof has a metric nature, we can conclude that every bounded sequence in a uniformly convex metric space has a regular subsequence.

Definition 2.5 [22] Let $K$ be a nonempty subset of a metric space $(X, d)$. A mapping $T: K \rightarrow X$ is said to satisfy condition (C) if for each $x, y \in K$,

$$
\frac{1}{2} d(x, T x) \leq d(x, y) \text { implies } d(T x, T y) \leq d(x, y) .
$$

In [23], Garcia-Falset et al. introduce two generalizations of condition (C) as follows.

Definition 2.6 Let $K$ be a nonempty subset of a metric space $(X, d)$. A mapping $t: K$ $\rightarrow X$ is said to satisfy condition $\left(C_{\lambda}\right)$ for some $\lambda \in(0,1)$ if for each $x, y \in K$,

$$
\lambda d(x, t(x)) \leq d(x, y) \text { implies } d(t(x), t(y)) \leq d(x, y) .
$$


Let $\mu \geq 1$. We say that $t: K \rightarrow X$ satisfies condition $\left(\mathrm{E}_{\mu}\right)$ if for each $x, y \in K$,

$$
d(x, t(y)) \leq \mu d(x, t(x))+d(x, y)
$$

We say that $t$ satisfies condition (E) if $t$ satisfies $\left(\mathrm{E}_{\mu}\right)$ for some $\mu \geq 1$.

Let $(X, d)$ be a geodesic space. We denote by $2^{X}$ the family of nonempty subsets of $X$, by $P(X)$ the family of nonempty proximinal subsets of $X$, by $K(X)$ the family of nonempty compact subsets of $X$, and by $K C(X)$ the family of nonempty compact convex subsets of $X$ : Let $H$ be the Hausdorff metric with respect to $d$, that is,

$$
H(A, B):=\max \left\{\sup _{a \in A} \operatorname{dist}(a, B), \sup _{b \in B} \operatorname{dist}(b, A)\right\}, \quad A, B \in 2^{X},
$$

where $\operatorname{dist}(a, B):=\inf \{d(a, b): b \in B\}$ is the distance from the point $a$ to the set $B$ : The metric projection (or nearest point mapping) $P_{B}$ onto $B$ is the mapping

$$
P_{B}(x)=\{b \in B: d(x, b)=\operatorname{dist}(x, B)\}, \text { for every } x \in X .
$$

We now state the multivalued analogs of conditions $(E)$ and $\left(C_{\lambda}\right)$ in the following manner:

Definition 2.7 A multivalued mapping $T: K \rightarrow 2^{X}$ is said to satisfy condition $\left(C_{\lambda}\right)$ for some $\lambda \in(0,1)$ if for each $x, y \in K$,

$$
\lambda \operatorname{dist}(x, T(x)) \leq d(x, y) \text { implies } H(T(x), T(y)) \leq d(x, y) .
$$

For $\mu \geq 1$. We say that $T$ satisfies condition $\left(\mathrm{E}_{\mu}\right)$ if for each $x, y \in K$,

$$
\operatorname{dist}(x, T(y)) \leq \mu \operatorname{dist}(x, T(x))+d(x, y) .
$$

We say that $T$ satisfies condition (E) if $T$ satisfies $\left(\mathrm{E}_{\mu}\right)$ for some $\mu \geq 1$.

There are other kinds of conditions $\left(C_{\lambda}\right)$ and $(E)$ for multivalued mappings defined by Razani and Salahifard [12] for the case of $\lambda>\frac{1}{2}$ in CAT(0) spaces.

They are studied in uniformly convex metric spaces by Espinola et al. [26]. We call them here the condition $\left(C_{\lambda}^{\prime}\right)$ (and (E') respectively.

Definition 2.8 A multivalued mapping $T: K \rightarrow 2^{X}$ is said to satisfy condition $\left(C_{\lambda}^{\prime}\right)$ if for each $x, y \in K$ and $u_{x} \in T(x)$ such that

$$
\lambda d\left(x, u_{x}\right) \leq d(x, y),
$$

there exists $u_{y} \in T(y)$ such that

$$
d\left(u_{x}, u_{y}\right) \leq d(x, y) .
$$

Let $\mu \geq 1$. We say that $T$ satisfies condition $\left(E_{\mu}^{\prime}\right)$ if for each $x, y \in K$ and $u_{x} \in T(x)$, there exists $u_{y} \in T(y)$ such that

$$
d\left(x, u_{y}\right) \leq \mu d\left(x, u_{x}\right)+d(x, y) .
$$

We say that $T$ satisfies condition (E') if $T$ satisfies $\left(E_{\mu}^{\prime}\right)$ for some $\mu \geq 1$.

We note from Definitions 2.7 and 2.8 that if $T$ takes compact values, then the condition $\left(C_{\lambda}\right)$ (resp. (E)) implies condition $\left(C_{\lambda}^{\prime}\right)$ (resp. $\left(E^{\prime}\right)$ ). On the other hand, Espinola et al. [26] prove that the condition $\left(C_{\frac{1}{2}}^{\prime}\right)$ implies condition $\left(E_{3}^{\prime}\right)$. By a slightly modification of the proof of Lemma 3.2 in [26], we can show that the condition $\left(\mathrm{C}_{\frac{1}{2}}\right)$ implies 
condition $\left(\mathrm{E}_{3}\right)$. However, it is unknown that condition $\left(C_{\lambda}^{\prime}\right)$ (resp. $\left(C_{\lambda}\right)$ ) implies condition (E') (resp. (E)) for $\lambda>\frac{1}{2}$ even in the case of single-valued mappings (see [[23], Remark 4]).

Recall that a single-valued mapping $t: K \rightarrow K$ and a multivalued mapping $T: K \rightarrow$ $2^{X}$ are said to be commuting ([33]) if $t(y) \in T(t(x))$ for all $x \in K$ and $y \in T(x)$. T and $t$ are said to be weakly commuting if $\left.t\left(\partial_{K} T(x)\right) \subseteq T(t(x))\right)$ for all $x \in K$, where $\partial_{K} A$ denotes the relative boundary of $A \subseteq K$. By using the fact that condition $\left(C_{\frac{1}{2}}^{\prime}\right)$ implies condition ( $\mathrm{E}_{3}^{\prime}$ ), Espinola et al. [26] ensure the existence of common fixed points for a pair of mappings $t$ and $T$ that are commuting and satisfying condition $\left(C_{\frac{1}{2}}^{\prime}\right)$ in uniformly convex metric spaces. We observe that their method can not be extended to the case of $\lambda>\frac{1}{2}$ because, in this case, we do not know if condition $\left(C_{\lambda}^{\prime}\right)$ implies condition (E'). However, if we assume, in addition, that the mapping $I-T$ is strongly demiclosed, then we can extend Espinola et al.'s result to the case of $\lambda>\frac{1}{2}$.

Definition 2.9 [23] Let $T: K \rightarrow 2^{X}$, we say that $I-T$ is strongly demi-closed if for every sequence $\left\{x_{n}\right\}$ in $K$ which converges to $x \in K$ and such that $\lim _{n} \operatorname{dist}\left(x_{n}, T\left(x_{n}\right)\right)=$ 0 , we have $x \in T(x)$.

Notice that for every continuous mapping $T: K \rightarrow 2^{X}, I-T$ is strongly demiclosed but the converse is not true (see [[23], Example 5]). The following proposition shows that condition (E') implies the strongly demiclosedness of $I-T$ but converse is not true (see [[23], Example 2]).

Proposition 2.10 Let $K \in 2^{X}$. If $T: K \rightarrow P(X)$ satisfies condition $\left(E^{\prime}\right)$ then $I-T$ is strongly demiclosed.

Proof. Let $\left\{x_{n}\right\}$ be a sequence in $K$ such that $\lim _{n} \operatorname{dist}\left(x_{n}, T(x n)\right)=0$ and $\lim _{n} x_{n}=x$ $\in K$. Since $T\left(x_{n}\right)$ is proximinal, we can choose $y_{n} \in T\left(x_{n}\right)$ such that

$$
d\left(x_{n}, y_{n}\right)=\operatorname{dist}\left(x_{n}, T\left(x_{n}\right)\right) .
$$

Since $T$ satisfies $\left(E_{\mu}^{\prime}\right)$ for some $\mu \geq 1$, there exists $z_{n} \in T(x)$ such that

$$
d\left(x_{n}, z_{n}\right) \leq \mu d\left(x_{n}, y_{n}\right)+d\left(x_{n}, x\right) .
$$

This implies

$$
\operatorname{dist}\left(x_{n}, T(x)\right) \leq \mu \operatorname{dist}\left(x_{n}, T\left(x_{n}\right)\right)+d\left(x_{n}, x\right) .
$$

By taking $n \rightarrow \infty$, we have $\lim _{n} \operatorname{dist}\left(x_{n}, T(x)\right)=0$. Thus

$$
\operatorname{dist}(x, T(x)) \leq d\left(x, x_{n}\right)+\operatorname{dist}\left(x_{n}, T(x)\right) \rightarrow 0 \text { as } n \rightarrow \infty .
$$

Therefore, $x \in T(x)$.

\section{Main results}

The following lemma is proved in [26] for the case of $\lambda>\frac{1}{2}$. As the same argument in the proof of [[26], Proposition 3.4], it is easy to see that the result also holds for $\lambda>\frac{1}{2}$.

Lemma 3.1 Let $X$ be a geodesic space with convex metric, $K$ be a bounded convex subset of $X$ and $T: K \rightarrow 2^{K}$. If $T$ satisfies condition $\left(C_{\lambda}^{\prime}\right)$,then $T$ has an approximate fixed point sequence in $K$ : 
Theorem 3.2 Let $X$ be a complete uniformly convex metric space and $K$ be a bounded closed convex subset of X: Suppose that $T: K \rightarrow K(K)$ satisfies condition $\left(C_{\lambda}^{\prime}\right)$ and $I-T$ is strongly demiclosed. Then $T$ has a fixed point.

Proof. By Lemma 3.1, $T$ has an approximate fixed point sequence in $K$, say $\left\{x_{n}\right\}$. By passing to a subsequence, we may suppose that $\left\{x_{n}\right\}$ is regular. Let $A\left(\left\{x_{n}\right\}\right)=\{x\}$. We will show that $x$ is a fixed point of $T$. Now, if $\lim _{\inf _{n}} d\left(x_{n}, x\right)=0$, again by passing to a subsequence, we may suppose that $\lim _{n} x_{n}=x \in K$. Since $I-T$ is strongly demiclosed, $x \in T(x)$. For the case that $\lim _{\inf _{n}} d\left(x_{n}, x\right)>0$, we let $\varepsilon=\frac{1}{2} \lim \inf _{n} d\left(x_{n}, x\right)$. For each $n \in \mathbb{N}$, we can choose $y_{n} \in T\left(x_{n}\right)$ such that

$$
d\left(x_{n}, y_{n}\right)=\operatorname{dist}\left(x_{n}, T\left(x_{n}\right)\right) .
$$

Notice that

$\lambda d\left(x_{n}, y_{n}\right)=\lambda d\left(x_{n}, T\left(x_{n}\right)\right)<\varepsilon<d\left(x_{n}, x\right)$ for sufficiently large $n \in \mathbb{N}$.

For such $n$, there exists $u_{n} \in T(x)$ such that $d\left(y_{n}, u_{n}\right) \leq d\left(x_{n}, x\right)$. Since $T(x)$ is compact, there exists a subsequence $\left\{u_{n_{k}}\right\}$ of $\left\{u_{n}\right\}$ such that $\lim _{k} u_{n_{k}}=u \in T(x)$. So

$$
\begin{aligned}
d\left(x_{n_{k}}, u\right) & \leq d\left(x_{n_{k}}, y_{n_{k}}\right)+d\left(y_{n_{k}}, u_{n_{k}}\right)+d\left(u_{n_{k}}, u\right) \\
& \leq d\left(x_{n_{k}}, T\left(x_{n_{k}}\right)\right)+d\left(x_{n_{k}}, x\right)+d\left(u_{n_{k}}, u\right) .
\end{aligned}
$$

This implies lim $\sup _{k} d\left(x_{n_{k}}, u\right) \leq \lim \sup _{k} d\left(x_{n_{k}}, x\right)$. Since $A\left(\left\{x_{n}\right\}\right)=\{x\}, x=u \in T(x)$.

Lemma 3.3 Let $K$ be a nonempty subset of a metric space $X$ and $t: K \rightarrow K$ be a quasi-nonexpansive mapping whose fixed point set is nonempty. Suppose that $T: K \rightarrow$ $2^{K}$ is such that for every $x, y \in F i x(t)$, the set $P_{\partial_{K} T(y)}(x)$ is a singleton. If $t$ and $T$ commute weakly, then $P_{\partial_{K} T(y)}(x) \in$ Fix $(t)$ for all $x, y \in$ Fix $(t)$.

Proof. Let $x, y \in F i x(t)$ and denote $P_{\partial_{K} T(y)}(x)$ by $u$. Since $t$ and $T$ commute weakly,

$$
t\left(\partial_{K} T(\gamma)\right) \subseteq T(t(\gamma))=T(\gamma) .
$$

Thus $t(u) \in T(y)$ : Hence

$$
d(x, t(u))=d(t(x), t(u)) \leq d(x, u) .
$$

Thus $t(u)=u$ and the conclusion follows.

Theorem 3.4 Let $X$ be a complete uniformly convex metric space, $K$ a bounded closed convex subset of $X$ and $t: K \rightarrow K$ a quasi-nonexpansive mapping whose fixed point set is nonempty. Suppose that $T: K \rightarrow K C(K)$ satisfies condition $\left(C_{\lambda}^{\prime}\right)$ and $I-T$ is strongly demiclosed. If $t$ and $T$ commute weakly, then there exists $z \in K$ such that $z=t(z) \in T(z)$.

Proof. Let $A=\operatorname{Fix}(t)$, then by Lemma 2.3, $A$ is closed and convex. By Proposition 2.2, the projection onto each compact and convex set is a singleton. By Lemma 3.3, $P_{\partial_{K} T(x)}(x) \in T(x) \cap A$ for all $x \in A$. We consider the mapping

$$
F(\cdot):=T(\cdot) \cap A: A \rightarrow K(A) .
$$

We show that $F$ satisfies condition $\left(C_{\lambda}^{\prime}\right)$ and $I-F$ is strongly demiclosed. Let and $x, y$ $\in A, u_{x} \in T(x) \cap A$ such that

$$
\lambda d\left(x, u_{x}\right) \leq d(x, y) .
$$

Since $T$ satisfies $\left(C_{\lambda}^{\prime}\right)$, there exists $v_{y} \in T(y)$ such that

$$
d\left(u_{x}, v_{y}\right) \leq d(x, y) .
$$


Let $u_{y}$ stands for $P_{\partial_{K} T(y)}\left(u_{x}\right)$. According to Lemma 3.3, $u_{y} \in T(y) \cap A$. It is also clear that

$$
d\left(u_{x}, u_{\gamma}\right) \leq d\left(u_{x}, v_{y}\right) \leq d(x, y) .
$$

Thus, $F$ satisfies $\left(C_{\lambda}^{\prime}\right)$. The strongly demiclosedness of $I-F$ follows from the one of $I$ - T. By Theorem 3.2, there exists $z \in K$ such that $z \in F(z)$ : Therefore $z=t(z) \in T(z)$.

It is known that if $t: K \rightarrow K$ has a fixed point, then both conditions $(\mathrm{E})$ and $\left(\mathrm{C}_{\lambda}\right)$ imply the quasi-nonexpansiveness of $t$. This fact yields the following corollary.

Corollary 3.5 Let $X$ be a complete uniformly convex metric space, $K$ a bounded closed convex subset of $X, t: K \rightarrow K$ a mapping satisfying condition $(E)$ or $\left(C_{\lambda}\right)$ for which Fix $(t)$ is nonempty. Suppose that $T: K \rightarrow K C(K)$ satisfies condition $\left(C_{\lambda}^{\prime}\right)$ and $I$ $T$ is strongly demiclosed. If $t$ and $T$ commute weakly, then there exists $z \in K$ such that $z=t(z) \in T(z)$.

By using the argument in the proof of Theorem 3.4, we can also obtain the following result that is an extension of [[4], Theorem 3.8]. Recall that a point $z \in X$ is said to be a center of the mapping $t: K \rightarrow X$ if for each $x \in K, d(z, t(x)) \leq d(z, x)$. The set of all centers of $t$ is denoted by $Z(t)$.

Theorem 3.6 Let $X$ be a complete uniformly convex metric space, $K$ a bounded closed convex subset of $X, t: K \rightarrow K$ a mapping. Suppose that $T: K \rightarrow K C(K)$ satisfies condition $\left(C_{\lambda}^{\prime}\right)$ and $I-T$ is strongly demiclosed. If $t$ and $T$ commute weakly, and $\varnothing \neq T(x) \cap$ Fix $(t) \subseteq Z(t)$, and Fix $(t)$ is closed and convex, then there exists $z \in K$ such that $z=t(z)$ $\in T(z)$.

Finally, we show that the strongly demiclosedness of $I-T$ in Theorems 3.2 and 3.4 is necessary.

Example 3.7 Put $X=\mathbb{R}$ and $K=[-1 / 4,1]$ : Let $t$ be the identity mapping on $K$ and let $T$ be the mapping on $K$ defined by

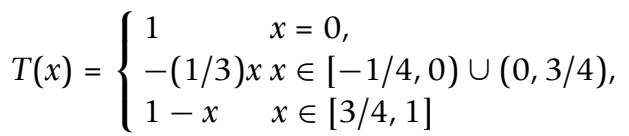

It is easy to see that $t$ and $T$ commute. In [23], the authors prove that either

$$
|T(x)-T(y)| \leq|x-y| \quad \text { or } \quad(3 / 4) \min \{|x-T(x)|,|y-T(y)|\} \geq|x-y| .
$$

We now let $\varepsilon \in\left(0, \frac{1}{4}\right)$, then either

$$
|T(x)-T(y)| \leq|x-y| \quad \text { or } \quad\left(\frac{3}{4}+\varepsilon\right) \min \{|x-T(x)|,|y-T(y)|\}>|x-y| .
$$

This implies that $T$ satisfies $\left(C_{\frac{3}{4}+\varepsilon}\right)$ for all $\varepsilon \in\left(0, \frac{1}{4}\right)$. Let $\left\{x_{n}\right\}=\left\{\frac{1}{n}\right\}$, then $\left\{x_{n}\right\}$ is an approximate fixed point sequence for $T$ which converges to 0 . But 0 is not a fixed point of $T$. This shows that $I-T$ is not strongly demiclosed. It is easy to see that $T$ does not have a fixed point. 


\section{Author details}

'Department of Mathematics, Faculty of Science, Chiang Mai University, Chiang Mai 50200, Thailand ${ }^{2}$ Centre of Excellence in Mathematics, CHE, Si Ayutthaya Rd., Bangkok 10400, Thailand

\section{Authors' contributions}

All authors read and approved the final manuscript.

\section{Competing interests}

The authors declare that they have no competing interests.

Received: 28 December 2010 Accepted: 25 July 2011 Published: 25 July 2011

\section{References}

1. Bridson, M, Haefliger, A: Metric Spaces of Non-Positive Curvature. Springer, Berlin (1999)

2. Kirk, WA: Geodesic geometry and fixed point theory. Seminar of Mathematical Analysis (Malaga/Seville, 2002/2003), pp. 195-225. Colecc. Abierta, 64, Univ. Sevilla Secr. Publ., Seville (2003)

3. Kirk, WA: Geodesic geometry and fixed point theory II. In: International Conference on Fixed Point Theory and Applications pp. 113-142. Yokohama Publ., Yokohama (2004)

4. Abkar, A, Eslamian, M: Common fixed point results in CAT(0) spaces. Nonlinear Anal TMA. 74, 1835-1840 (2011). doi:10.1016/j.na.2010.10.056

5. Chaoha, P, Phon-on, A: A note on fixed point sets in CAT(0) spaces. J Math Anal Appl. 320, 983-987 (2006). doi:10.1016/ j.jmaa.2005.08.006

6. Dhompongsa, S, Kaewkhao, A, Panyanak, B: Lim's theorems for multivalued mappings in CAT(0) spaces. J Math Anal Appl. 312, 478-487 (2005). doi:10.1016/j.jmaa.2005.03.055

7. Espinola, R, Fernandez-Leon, A: CAT(k)-spaces, weak convergence and fixed points. J Math Anal Appl. 353, 410-427 (2009). doi:10.1016/j.jmaa.2008.12.015

8. Espinola, R, Hussain, N: Common fixed points for multimaps in metric spaces. Fixed Point Theory Appl. 2010, Article ID 204981, 1-14 (2010)

9. Hussain, N, Khamsi, MA: On asymptotic pointwise contractions in metric spaces. Nonlinear Anal TMA. 71, 4423-4429 (2009). doi:10.1016/.jna.2009.02.126

10. Khan, SH, Abbas, M: Strong and $\triangle$-convergence of some iterative schemes in CAT(0) spaces. Comput Math Appl. 61, 109-116 (2011). doi:10.1016/j.camwa.2010.10.037

11. Leustean, L: A quadratic rate of asymptotic regularity for CAT(0)-spaces. J Math Anal Appl. 325, 386-399 (2007). doi:10.1016/j.jmaa.2006.01.081

12. Razani, A, Salahifard, H: Invariant approximation for CAT(0) spaces. Non-linear Anal TMA. 72, 2421-2425 (2010). doi:10.1016/j.na.2009.10.039

13. Saejung, S: Halpern's iteration in CAT(0) spaces. Fixed Point Theory Appl. 2010, Article ID 471781, 1-13 (2010)

14. Shahzad, N, Markin, J: Invariant approximations for commuting mappings in CAT(0) and hyperconvex spaces. J Math Anal Appl. 337, 1457-1464 (2008). doi:10.1016/j.jmaa.2007.04.041

15. Bartolini, I, Ciaccia, P, Patella, M: String Matching with Metric Trees Using an Approximate Distance. SPIR Lecture Notes in Computer Science, vol. 2476. Springer, Berlin (1999)

16. Bestvina, M: R-trees in topology, geometry, and group theory. In: Handbook of Geometric Topology, pp. 55-91. NorthHolland, Amsterdam (2002)

17. Espinola, R, Kirk, WA: Fixed point theorems in R-trees with applications to graph theory. Topol Appl. 153, 1046-1055 (2006). doi:10.1016/.jtopol.2005.03.001

18. Kirk, WA: Fixed point theorems in CAT(0) spaces and R-trees. Fixed Point Theory Appl. 2004, 309-316 (2004). doi:10.1155/S1687182004406081

19. Park, S: The KKM principle in abstract convex spaces: equivalent formulations and applications. Nonlinear Anal TMA. 73, 1028-1042 (2010). doi:10.1016/j.na.2010.04.029

20. Semple, C, Steel, M: Phylogenetics. Oxford Lecture Ser. Math Appl, vol. 24, Oxford University Press, Oxford (2003)

21. Shahzad, N: Fixed point results for multimaps in CAT(0) spaces. Topol Appl. 156, 997-1001 (2009). doi:10.1016/j. topol.2008.11.016

22. Suzuki, T: Fixed point theorems and convergence theorems for some generalized nonexpansive mapping. J Math Anal Appl. 340, 1088-1095 (2008). doi:10.1016/j.jmaa.2007.09.023

23. Garcia-Falset, J, Lorens-Fuster, E, Suzuki, T: Fixed point theory for a class of generalized nonexpansive mappings. J Math Anal Appl. 375, 185-195 (2011). doi:10.1016/j.jmaa.2010.08.069

24. Goebel, K, Reich, S: Uniform Convexity, Hyperbolic Geometry, and Nonexpansive Mappings. Marcel Dekker, Inc. New York (1984)

25. Abkar, A, Eslamian, M: Fixed point theorems for Suzuki generalized nonexpansive multivalued mappings in Banach spaces. Fixed Point Thoery Appl. 2010, Article ID 457935, 1-10 (2010)

26. Espinola, R, Lorenzo, P, Nicolae, A: Fixed points, selections and common fixed points for nonexpansive-type mappings. J Math Anal Appl. 382, 503-515 (2011). doi:10.1016/j.jmaa.2010.06.039

27. Kaewcharoen, A, Panyanak, B: Fixed point theorems for some generalized multivalued nonexpansive mappings. Nonlinear Anal TMA. 74, 5578-5584 (2011). doi:10.1016/..na.2011.05.042

28. Kohlenbach, U, Leustean, L: Asymptotically nonexpansive mappings in uniformly convex hyperbolic spaces. J Eur Math Soc. 12, 71-92 (2010)

29. Shimizu, T, Takahashi, W: Fixed point theorems in certain convex metric spaces. Math Japonica. 37, 855-859 (1992)

30. Kaewcharoen, A, Panyanak, B: Fixed points for multivalued mappings in uniformly convex metric spaces. Int J Math Math Sci. 2008, Article ID 163580, 1-9 (2008)

31. Espinola, R, Fernandez-Leon, A, Piatek, B: Fixed points of single- and setvalued mappings in uniformly convex metric spaces with no metric convexity. Fixed Point Theory Appl. 2010, Article ID 169837, 1-16 (2010) 
32. Goebel, K, Kirk, WA: Topics in Metric Fixed Point Theory. Cambridge Univ. Press, Cambridge (1990)

33. Itoh, S, Takahashi, W: The common fixed point theory of singlevalued mappings and multivalued mappings. Pacific J Math. 79, 493-508 (1978)

doi:10.1186/1687-1812-2011-20

Cite this article as: Laowang and Panyanak: Common fixed points for some generalized multivalued nonexpansive mappings in uniformly convex metric spaces. Fixed Point Theory and Applications 2011 2011:20.

Submit your manuscript to a SpringerOpen ${ }^{\odot}$ journal and benefit from:

- Convenient online submission

- Rigorous peer review

- Immediate publication on acceptance

- Open access: articles freely available online

- High visibility within the field

- Retaining the copyright to your article

Submit your next manuscript at $\boldsymbol{\nabla}$ springeropen.com 